\title{
BMJ Open Activities outside of the care setting for people with dementia: a systematic review
}

\author{
Nathan Martin D'Cunha (D) , ${ }^{1,2}$ Stephen Isbel, ${ }^{1}$ Andrew J McKune, ${ }^{1,3}$ Jane Kellett, ${ }^{1,2}$ \\ Nenad Naumovski (1) ${ }^{1,2}$
}

To cite: D'Cunha NM, Isbel S, McKune AJ, et al. Activities outside of the care setting for people with dementia: a systematic review. BMJ Open 2020;10:e040753. doi:10.1136/ bmjopen-2020-040753

- Prepublication history for this paper is available online. To view these files, please visit the journal online (http://dx.doi. org/10.1136/bmjopen-2020040753).

Received 21 May 2020 Revised 20 July 2020 Accepted 04 August 2020

\section{Check for updates}

C Author(s) (or their employer(s)) 2020. Re-use permitted under CC BY-NC. No commercial re-use. See rights and permissions. Published by BMJ.

${ }^{1}$ School of Rehabilitation and Exercise Sciences, Faculty of Health, University of Canberra, Bruce, Australian Capital Territory, Australia

${ }^{2}$ Functional Foods and Nutrition Research (FFNR) Laboratory, University of Canberra, Bruce, Australian Capital Territory, Australia

${ }^{3}$ Discipline of Biokinetics, Exercise and Leisure Sciences, School of Health Sciences, University of KwaZulu-Natal, Durban, South Africa

Correspondence to Dr Nenad Naumovski; nenad.naumovski@canberra. edu.au

\section{ABSTRACT}

Objectives To summarise the evidence from interventions investigating the effects of out of care setting activities on people with dementia living in residential aged care.

Design A systematic review.

Methods A systematic search of electronic databases (PubMed, PsycINF0, Scopus, Web of Science and the Cochrane Library) was performed to identify intervention trials published from journal inception to January 2020. Controlled trials, or quasi-experimental trials, which measured pre-intervention, post-intervention or duringintervention outcomes, where the participants were required to leave the care setting to participate in an intervention, were eligible for inclusion. Quality appraisal of the studies was performed following the Cochrane Collaboration's Risk of Bias or Newcastle-0ttawa Scale tools.

Results of the 4155 articles screened, 11 articles met the inclusion criteria from 9 different studies. The number of participants in the studies ranged from 6 to 70 people living with dementia and lasted for 3 weeks up to 5 months. The interventions were aquatic exercise, wheelchair cycling, art gallery discussion groups, an intergenerational mentorship programme, horse riding, walking and outdoor gardening. Overall, the studies indicated preliminary evidence of psychological $(n=7)$, physical $(n=4)$ and physiological $(n=1)$ benefits, and all interventions were feasible to conduct away from the aged care facilities. However, the low number of participants in the included studies $(n=177)$, the absence of a control group in all but three studies, and potential for selection bias, limits the generalisability of the findings.

Conclusions Activities outside of the residential aged care setting have the potential to be effective at providing a range of benefits for people living with dementia. Higher quality studies are required to encourage care providers to implement these type of activities in dementia care settings.

PROSPERO registration number CRD42020166518.

\section{BACKGROUND}

A high proportion of people living in residential aged care facilities (RACF) (also known as nursing homes, long-term care, care homes and assisted living among others) are living with dementia. Generally, people living with dementia in RACF have more progressive

\section{Strengths and limitations of this study}

To the best of our knowledge, this is the first systematic review to examine the effects of activities outside of the care setting in people with dementia.

- The different types of interventions with quantitative results are summarised, appraised and their benefits discussed.

- Only 9 different interventions from 11 publications were identified, highlighting a need for more research in this area.

dementia and greater physical limitations than those living in the community and often require high levels of care. ${ }^{1}$ As such, there is increasing recognition of the importance of providing high-quality, 24-hour care in RACF and dementia care settings. While the quality of care and the care environment is undoubtedly critical to health outcomes, access to meaningful activities and leisure in these settings can enhance physical functioning and cognitive and mental health. ${ }^{2}$ Person-centred care is based on respecting the values and priorities of the person and in RACF as it promotes quality of life, dignity and autonomy. Psychosocial interventions and meaningful activities for people with dementia can form an important part of person-centred care and include engagement with art and music, use of assistive technology, life story work and horticultural therapy. ${ }^{2}$

Previous studies have indicated that psychosocial interventions provide positive effects on mood, apathy and have the potential to reduce depression in people living in RACF. ${ }^{3}$ With the exception of horticultural therapy, which typically occurs outdoors but on the premises of RACF, these activities commonly occur indoors. Several reviews have evaluated the effects of psychosocial interventions across community and RACF settings which promote beneficial psychological, physical and cognitive effects. ${ }^{2-5}$ However, to the best 
of our knowledge, there has not been a consolidation of the evidence relating to the effects of participating in meaningful activities outside the RACF setting, on people living with dementia. Although it is established that being outdoors reduces stress and anxiety in people with dementia, ${ }^{6}$ safe and regular access to meaningful activities outside of the RACF are typically not possible without assistance from family or care staff. ${ }^{7}$ Recently, connecting people to their community and the outdoors is becoming an increased focus of aged care models as part of personcentred care principles and dementia-friendly communities. Activities outside of the care setting for older people and people with dementia have the potential to enable them to engage with the broader community, and have sense of community, contribution, belonging and social connectedness. These activities may also be activities they engaged in before moving to the RACF. Therefore, we systematically examined the literature which tested psychosocial interventions that enabled people living with dementia to leave the care setting to participate in activities outside of the RACF. The findings of this review will provide clarification to the current evidence base, with the aim to determine which interventions are the most effective in promoting well-being in this population as well as identify priority areas for future research.

\section{METHODS}

\section{Protocol and registration}

Methods for this systematic review followed the checklist from the Preferred Reporting Items for Systematic Reviews and Meta-Analyses (PRISMSA) guidelines. ${ }^{8}$ Patients and the public were not involved in the design, conduct, reporting or dissemination of this research.

\section{Selection criteria}

All comparative quantitative intervention studies, excluding case studies, were included. A PICOS (population, intervention, comparator, outcomes and setting) approach was used to guide the structure of the systematic review:

- Population: human participants living in RACF (also referred to as long-term care, nursing home, care home and residential care) with any form of dementia or cognitive impairment.

- Intervention: controlled trials, or quasi-experimental trials, which measured pre-intervention, postintervention or during-intervention outcomes, where the participants left the RACF setting to participate in the intervention.

- Comparator: using an active control condition, usual care or comparing pre-intervention and postintervention outcomes.

- Outcomes: any quantitative methods evaluating participant outcomes (directly or indirectly), which measure psychological health and well-being.

- Setting: participants must be living in the RACF.

\section{Search strategy}

Electronic database searches of PubMed, PsycINFO, Scopus, Web of Science and the Cochrane Library were performed for articles published from database inception to January 2020. The keyword search was: dement* OR alzheimer* OR 'cognitive impairment' AND psychosocial OR non-pharmacological OR 'leisure activities' OR 'activity programme' OR outdoor* OR outside OR horticultur* OR museum OR gallery OR music OR dance OR choir OR cafe OR theatre OR theatre OR film OR movie* OR 'meaningful activity' OR 'occupational therapy' AND 'aged care' OR institution* OR 'nursing home' OR 'long term care' OR 'care home' OR 'residential care' (online supplemental file). The reference lists of all included studies, and related existing systematic reviews, were searched for additional relevant articles. The searches were re-run prior to finalisation of the data analysis on the 15 May 2020.

\section{Study selection and data extraction}

Eligible studies met the following criteria: use a controlled study design, or compared pre-intervention and postintervention effects; involved human participants of any age living in RACF with dementia or cognitive impairment; and quantitatively examined the effects of an intervention which required participants to leave the RACF setting. Studies were excluded if they were epidemiological or qualitative studies, reviews, abstracts, conference papers or study protocols.

Two authors (NMD and NN) independently performed the searches using Covidence systematic review software (Veritas Health Innovation, Melbourne, Australia). Following the removal of duplicates, articles were excluded based on title and abstract screening using the selection criteria. For articles where this was unclear, the article was carried forward into the full-text review. The full-text of each article was read and assessed by the same authors, and independently evaluated to determine if all selection criteria were met. The authors met to discuss each article, and a third author (SI) was present to resolve disagreements.

A customised data extraction form was used to enter data from each article by one author (NMD) and crosschecked by a second author (SI). This included the aims, study design, description of the intervention, details of selection criteria (eg, severity of dementia (where applicable), age and level of care), outcomes and measures, and results. For included studies that used mixedmethods, only the quantitative results were presented and discussed. In addition, if studies included people with dementia living in RACF and in the community, only the data from the participants in the RACF were presented where possible. The results of the included studies were synthesised quantitatively and qualitatively.

\section{Quality assessment}

Due to the broad inclusion criteria, two risk of bias tools were used independently by two researchers 
(NMD and SI). For randomised controlled trials, the Cochrane Collaboration's Risk of Bias tool was used. ${ }^{9}$ The criteria for this tool assesses risk of bias for sequence generation, allocation concealment, blinding of participants, personnel and outcome assessors, incomplete outcome data, and selective outcome reporting. For each criterion, studies were assessed for risk of bias as low, unclear or high. For non-randomised and quasi-experimental studies, the Newcastle-Ottawa Scale (NOS) for non-randomised studies was used for the quality assessment. ${ }^{10}$ The NOS assesses methodological quality based on participant selection, comparability of treatment groups and outcome ascertainment with a maximum score of 9. Currently, there is no recommended tool to assess quality for quasiexperimental studies with only a treatment group, the highest possible score for these studies was 6 as previously described. ${ }^{11}$
RESULTS

\section{Study selection}

The systematic review search resulted in 6453 records for screening and 2308 duplicates were removed. Ten articles were identified through searching of the reference lists of relevant systematic reviews and included articles. Following title and abstract screening, 173 references remained for full-text review. In total, 162 records were excluded. In order, the articles were excluded because they failed the inclusion criteria for the following reasons: not published in English $(\mathrm{n}=4)$, not a journal article (conference abstract or not peer reviewed) $(n=12)$, wrong study design (qualitative study or survey) $(n=11)$, wrong population (participants not living with dementia, or participants not living in RACF) $(\mathrm{n}=20)$ and wrong intervention (intervention did not require participants to leave the RACF $(\mathrm{n}=114)$. One study was excluded as we were unable to receive clarification from the authors as

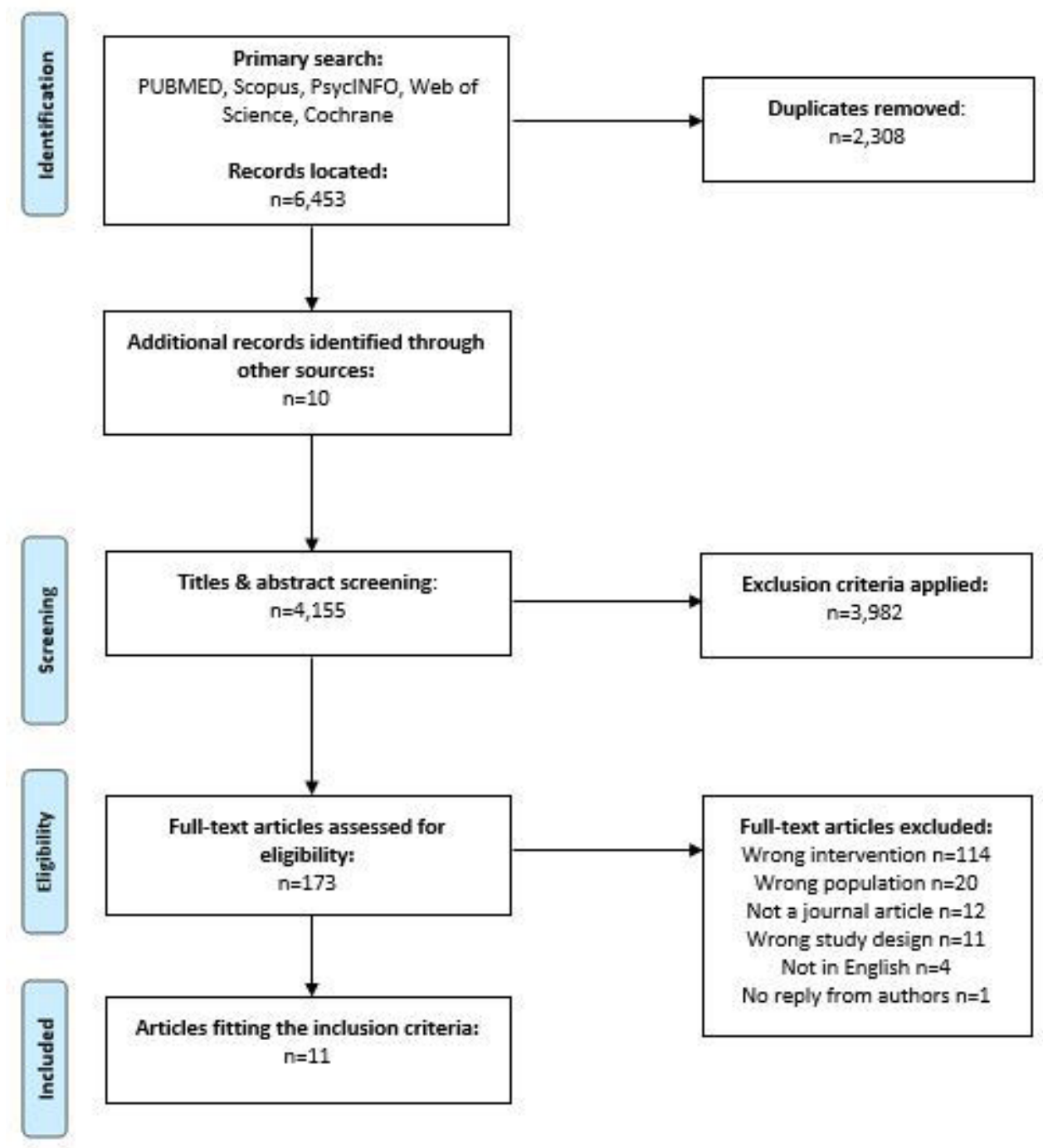

Figure 1 Preferred Reporting Items for Systematic Reviews and Meta-Analyses flow chart summary of systematic review search process. 
to whether participants left the RACF for the intervention. Eleven articles reporting on nine different studies fit the inclusion criteria. ${ }^{12-22}$ The PRISMSA flow chart is presented in figure 1. A meta-analysis was not performed due to the diversity of the included interventions and the reported outcome measures.

\section{Study characteristics}

Of the 11 included articles, ${ }^{12-22} 4$ reported on 2 different studies. Therefore, a total of nine studies were included. The 9 studies reported on data from a total of 177 participants living in RACF from high-income countries, Australia, ${ }^{1417-21}$ the USA ${ }^{12131516}$ and Sweden ${ }^{22}$ (table 1). Study sample sizes ranged from ${ }^{15} 6$ to $70 .{ }^{13}$ Two studies were randomised controlled trials, ${ }^{12}{ }^{13} 16$ one study was a controlled trial ${ }^{17}$ and the remaining six studies used quasiexperimental designs. ${ }^{14} 15$ 18-22 The participants ranged in age from 77.0 years $^{19}$ to 88.4 years. $^{1821}$

\section{Interventions}

Three of the articles included people living with dementia participating in a swimming-based programme at a local pool. ${ }^{171821}$ Two of these articles were from the same pilot study. ${ }^{182}$ Two articles were from one study of a recreational programme which included wheelchair cycling. ${ }^{13}$ Two studies were of arts-based discussion at an art gallery. ${ }^{1420}$ The remaining studies were horse riding (equine-assisted activities),$^{15}$ mentorship visits as part of an intergenerational programme, ${ }^{16}$ gardening at a park adjacent to the RACF ${ }^{22}$ and supervised walks in the community. ${ }^{19}$ The duration of the interventions lasted from two weeks ${ }^{12}$ to five months. ${ }^{16}$

\section{Outcome measures}

Four articles assessed behavioural and psychological symptoms, including agitation. ${ }^{12} 131721$ Four articles assessed depressive symptoms. ${ }^{13141617}$ Four articles assessed physical function ${ }^{14171822}$ and one study evaluated quantitative characteristics surrounding walks outside of the RACF, including the number of walks taken and reasons for walks not proceeding. ${ }^{19}$ Two studies each assessed cognitive function ${ }^{1416}$ and anxiety. ${ }^{16}{ }^{17}$ One study assessed cognitive function at baseline to determine if it affected participation in gardening activities. ${ }^{22}$ One article assessed selfreported quality of life, ${ }^{14}$ and one used an observer-rated quality of life instrument. ${ }^{15}$ One article each assessed changes in the number of sleep disturbances ${ }^{13}$ physiological outcomes (salivary cortisol and interleukin-6) ${ }^{14}$ and activities of daily living. ${ }^{17}$ During the intervention, four articles assessed changes in activity participation and engagement, ${ }^{12131520}$ and one article rated well-being. ${ }^{14}$

\section{Quality assessment}

The selection criteria allowed the inclusion of randomised studies, non-randomised studies and quasi-experimental studies. Therefore, the included studies were mostly preliminary by study design and did not represent conclusive evidence of the efficacy of the interventions. Two randomised controlled trials were evaluated using the
Cochrane risk of bias tool (table 2). ${ }^{12} 1316$ Two publications (one study) about wheelchair cycling were rated as most unclear, and high risk of bias for allocation concealment. ${ }^{12} 13$ The wheelchair cycling studies and the study by George nd Singer were rated with high risk of bias for blinding of personnel and participants. ${ }^{12} 1316$ However, the study by George and Singer was rated low or unclear for the other criteria. The remaining studies were assessed using the Newcastle-Ottawa Quality Assessment for nonrandomised studies. Only the study by Henwood et al was scored out of 9 due to the presence of a control group $(8 / 9) .{ }^{17}$ The six other studies were of good quality $(4 / 6-$ $5 / 6)$ (table 3). ${ }^{14}{ }^{15}{ }^{18-22}$ All six of these studies received a score of 0 for 'outcome assessment' as they were not independent blind assessments. Two studies did not recruit representative samples. ${ }^{1522}$ The study by Fields $e t$ al recruited participants with horse riding experience, ${ }^{15}$ and the study by Thelander $e t a l$ selected participants with a documented history of agitated or restless behaviour. ${ }^{22}$ The first swimming study lost greater than $25 \%$ of participants due to attrition and was rated 0 in the 'adequate cohort follow-up category'. ${ }^{18} 21$

\section{Narrative synthesis of intervention effects}

Three articles from two studies by the same research team investigated the effects of twice weekly participation in the Watermemories Swimming Club. ${ }^{17} 1821$ The programme aimed to create positive memories in people who previously enjoyed swimming. The pilot study results were reported in two publications by Neville et al and Henwood et al with 11 people with dementia recruited from 2 RACF. ${ }^{181}$ The 12-week intervention incorporated supervised aquatic exercise at a local municipal pool and was designed to promote physical and functional ability. The study by Neville et al reported outcome measures of psychological well-being. ${ }^{21}$ The Psychological Well-Being in Cognitively Impaired Persons Scale (PWBCIP) and the Revised Memory and Behaviour Problems Checklist (RMBPC) were used to assess positive and negative affective states and engagement, and behavioural and psychological symptoms of dementia (BPSD), respectively. Data were collected at baseline, week 6, week 9 and post-intervention. Across timepoints, improvements were observed in the PWB-CIP ( $n=8 ; p=0.034)$, RMBPC (BPSD frequency) and RMBPC (staff reaction to BPSD) (both: $\mathrm{n}=10 ; \mathrm{p}=0.001$ ). The study by Henwood et al focused on physical and functional benefits of the Watermemories Swimming Club. ${ }^{18}$ The outcome measures were hand grip muscle strength and body composition, with balance and functional capacity measured by two composite measures, the Balance Outcome Measure for Elder Rehabilitation and the Short Physical Performance Battery. Of the 0 participants who provided data, an improvement in left-hand grip strength was observed $(p=0.017)$. Both positive and negative non-significant trends were observed for other outcomes. However, transportation of people with dementia from the RACF to a swimming pool was considered feasible and set the foundation for the 


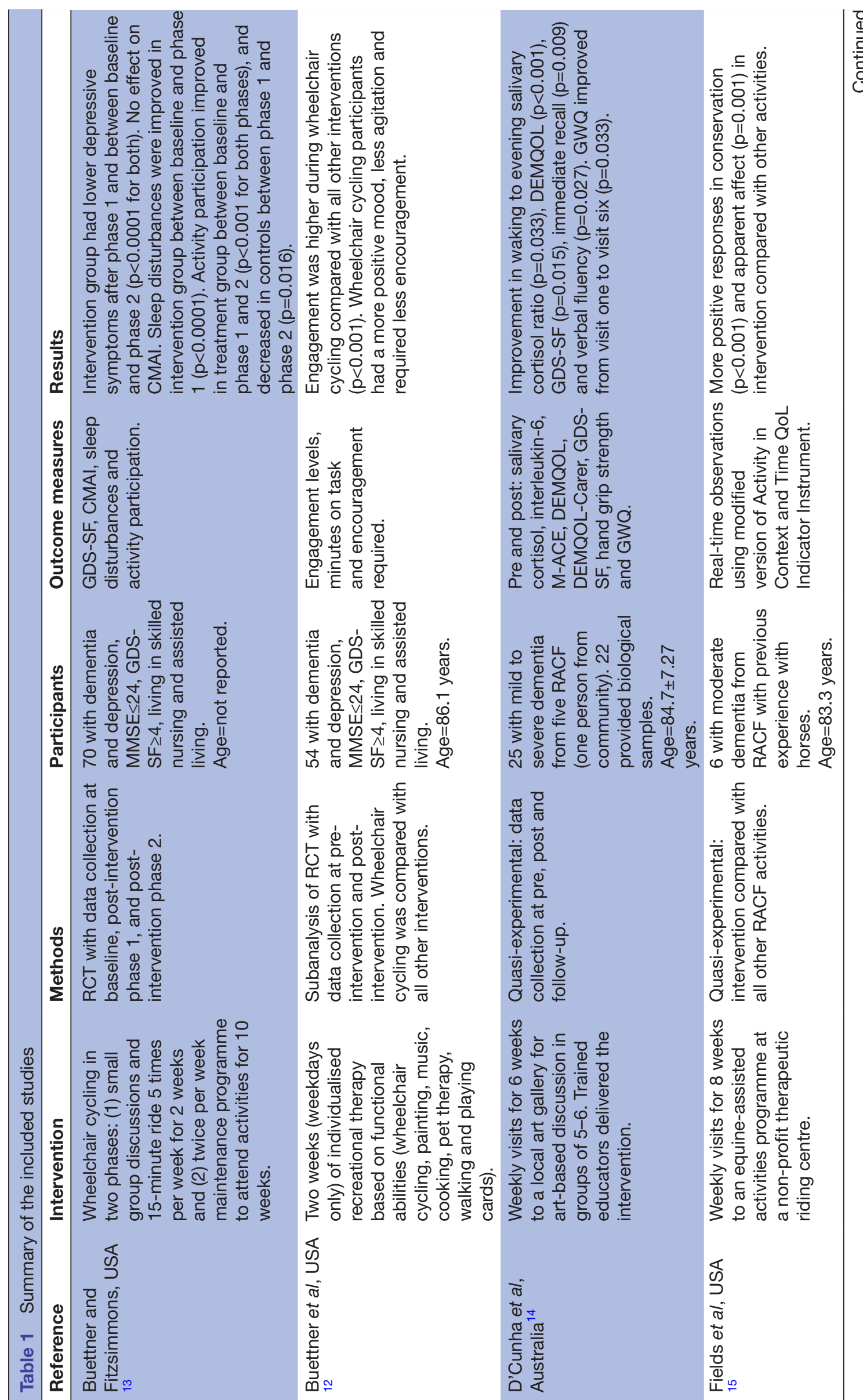




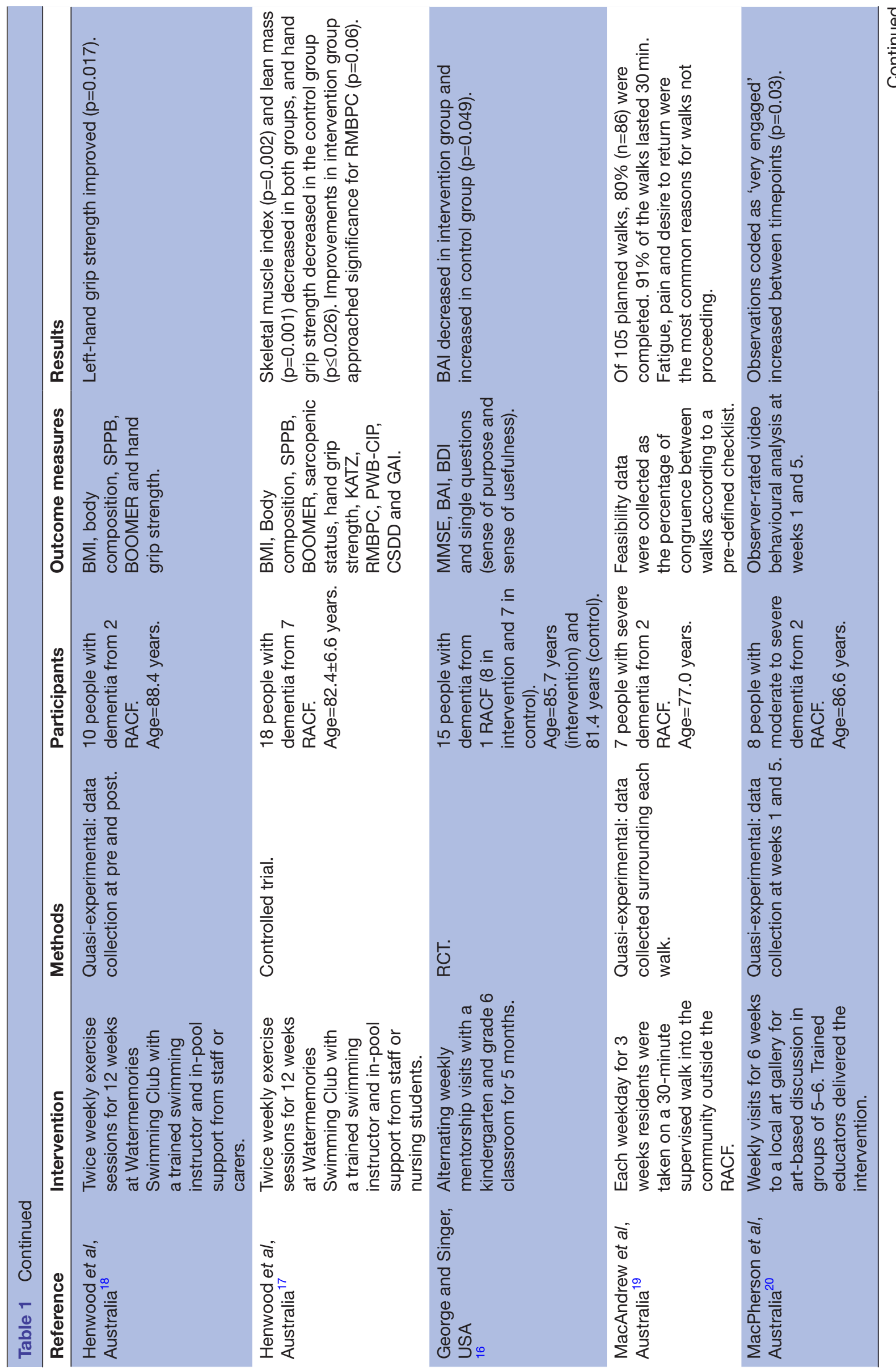



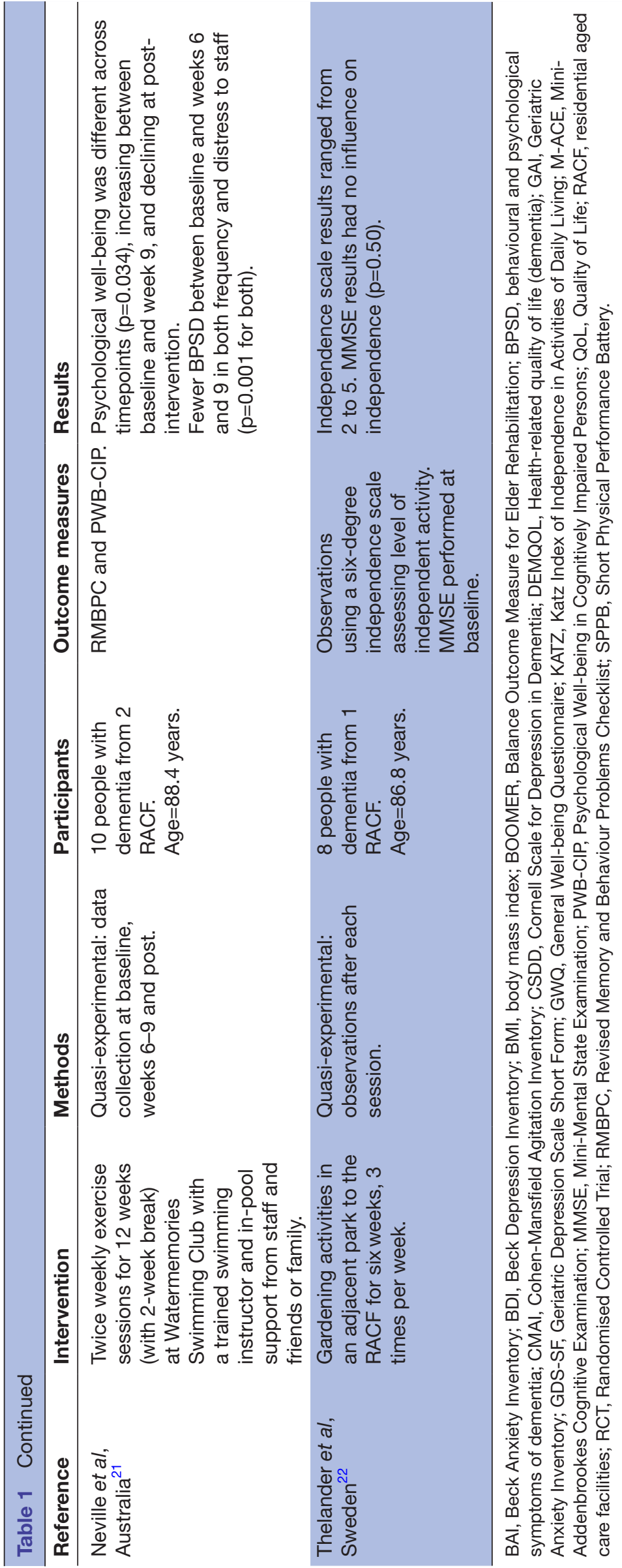
Table 2 Quality assessment of included randomised studies

\begin{tabular}{|c|c|c|c|c|c|c|c|}
\hline & $\begin{array}{l}\text { Random } \\
\text { sequence } \\
\text { generation }\end{array}$ & $\begin{array}{l}\text { Allocation } \\
\text { concealment }\end{array}$ & $\begin{array}{l}\text { Blinding of } \\
\text { participants } \\
\text { and personnel }\end{array}$ & $\begin{array}{l}\text { Blinding of } \\
\text { outcome } \\
\text { assessment }\end{array}$ & $\begin{array}{l}\text { Incomplete } \\
\text { outcome data }\end{array}$ & $\begin{array}{l}\text { Selective } \\
\text { reporting }\end{array}$ & $\begin{array}{l}\text { Other } \\
\text { bias }\end{array}$ \\
\hline $\begin{array}{l}\text { Buettner et } a l^{13} \\
\text { and Buettner et } \\
a l^{12}\end{array}$ & Unclear & High & High & Unclear & Unclear & Unclear & Unclear \\
\hline George et $a l^{16}$ & Low & Unclear & High & Unclear & Low & Low & Unclear \\
\hline
\end{tabular}

controlled trial by Henwood et al. ${ }^{17}$ This study was also a 12-week intervention, but included a control group. The outcome measures were the same as the pilot study, ${ }^{18} 21$ but also included the Cornell Scale for Depression in Dementia, Geriatric Anxiety Inventory, KATZ Activities of Daily Living Scale and sarcopenia status using the European Working Group in Older People criteria. Of the two RACF involved in this study, one had considerably better compliance $(87.5 \%$ vs $44.6 \%)$. The analytic sample consisted of nine participants in both the intervention and control groups. Both groups had a decrease in Skeletal Mass Index and lean mass post-intervention ( $\mathrm{p}=0.002$ and $\mathrm{p}=0.001$, respectively). A significant group by time interaction was observed for left-hand and right-hand grip strengths $(\mathrm{p}=0.017$ and $\mathrm{p}=0.003$, respectively $)$, driven by large decreases in the control group $(\mathrm{p} \leq 0.026)$. Sarcopenia prevalence was increased post-intervention in both groups compared with baseline, increasing from four to six participants in the intervention group $(p=0.038)$ and from one to all nine participants in the control group $(p=0.002)$. Greater improvements were observed in the intervention group in the behavioural and psychological outcomes and activities of daily living, but these were non-significant (all, $\mathrm{p}>0.05$ ). Overall, the results of these studies reveal that away from the RACF, swimming is feasible, although both studies had participant attrition.

Wheelchair cycling was the intervention in two articles from one study of recreational therapy activities. ${ }^{12} 13$ In this activity, people with dementia and depressive symptoms sat in a wheelchair attached to a half bicycle and receive a 15-minute ride, where conversation with the cyclist was encouraged. An intensive 2-week programme with daily rides on the weekdays was first implemented with 41 and 29 participants from 2 RACF (total, $n=70$ ). This was followed by a 10-week maintenance period, where each participant went for a ride twice per week. The primary outcome in the first study was change in depressive symptoms assessed using the Geriatric Depression Scale Short Form. ${ }^{13}$ The intervention group had reduced depressive symptoms between baseline and after the intensive 2-week programme $(\mathrm{p}<0.001)$ and between baseline and after the 10-week maintenance programme $(\mathrm{p}<0.001)$. Depressive symptoms in the control group increased between baseline and by the end of the intensive 2-week programme $(p=0.047)$. Sleep disturbances were also recorded in one of the RACF participating in the study $(n=41)$. At baseline, sleep disturbances were reported in five participants in the intervention group and four in the control group. After the intensive 2-week programme, sleep disturbances were reported in two people in the treatment group and nine in the control group $(\mathrm{p}<0.001)$. Activity participation increased in the intervention group between baseline and following the intensive 2-week programme $(p<0.001)$, and in the maintenance programme $(\mathrm{p}<0.001)$. Between baseline and the end of the maintenance programme, the control group was participating in fewer activities $(p=0.016)$. The second study compared the amounts of encouragement and engagement, agitation and mood levels in a subsample of 54 participants who received the wheelchair biking during the intensive 2-week programme with the 72 other recreational activities that were carried out over the 2 weeks. ${ }^{12}$ These activities included playing cards, painting, music, pet therapy and walking. Participants spent longer participating in wheelchair cycling $(\mathrm{p}<0.001)$. Less encouragement, higher mood, lower agitation and greater engagement were all observed for wheelchair cycling compared with the other activities. Only the effect for engagement was statistically significant $(p<0.001)$. The results of these two studies demonstrated benefits from being outside participating in wheelchair cycling in people with dementia and depression in RACF.

Two studies of the National Gallery of Australia Art and Dementia programme met the inclusion criteria. ${ }^{1420}$ In both studies, each with an intervention period of 6 weeks, participants who were living in RACF were transported to the art gallery by bus and were accompanied by care staff. Most participants were recruited because care staff believed they would benefit from the programme. The art and dementia programme at the National Gallery of Australia is similar to the programme pioneered by the Museum of Modern Art in New York City. ${ }^{23}$ Typically, participants discuss and engage with artworks from the galleries collection during a 1-hour pre-planned tour. The study by MacPherson et al recruited 15 people living with dementia, 8 from a single RACF. ${ }^{20}$ Prior to the study, participants were reported to manifest significant challenging behaviours in their daily life. Observer-rated video analysis of the weeks 1 and 5 visits to the art gallery revealed an increase in observations coded as 'very engaged' $(p=0.03)$. Two-threeweeks after the final visit, one participant was able to clearly remember the programme, while others remembered aspects when prompted, and two did not remember. The study by D'Cunha et al at the same 


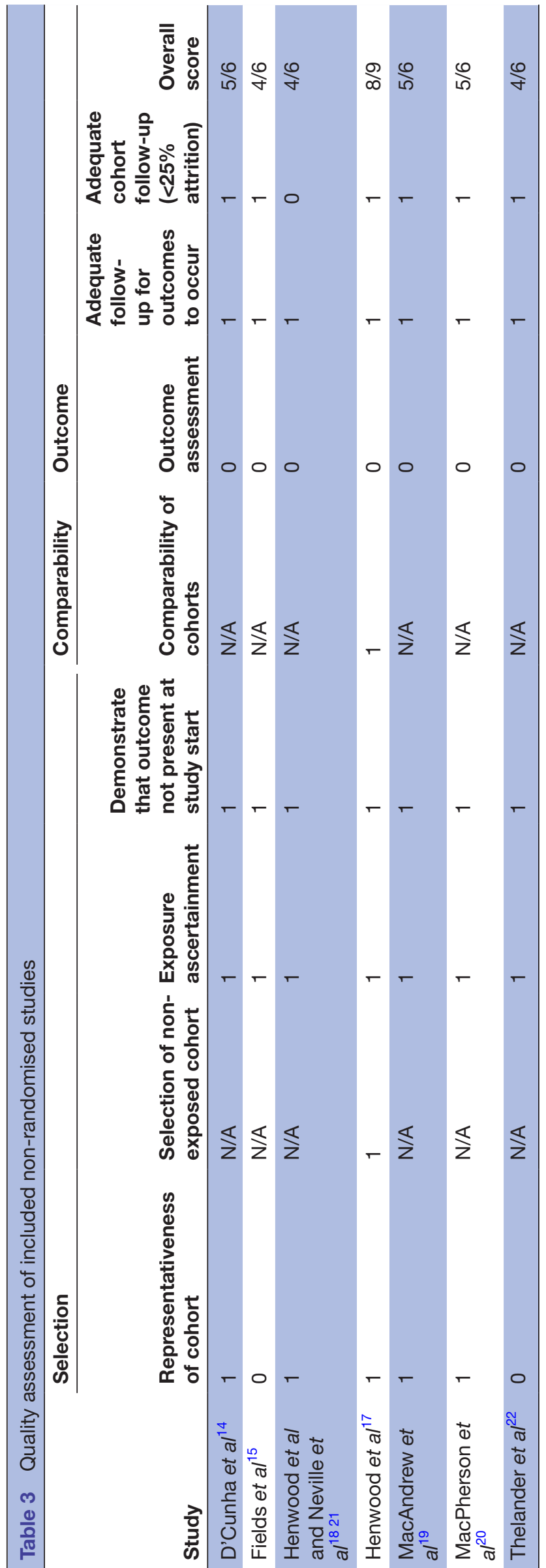

art gallery recruited 28 participants and 25 completed pre-intervention and post-intervention, and follow-up outcome measures (quality of life, depressive symptoms and cognitive performance). ${ }^{14}$ Of the 25 participants, 22 were living full time in RACF. Improvements were observed in each of the outcomes (all, $\mathrm{p}<0.05$ ), except for proxy-rated quality of life $(p=0.076)$. Unique to the literature on art gallery programmes for people living with dementia, this study also evaluated changes in salivary cortisol and interleukin- $6(\mathrm{n}=22)$, and hand grip strength. The waking to evening salivary cortisol ratio improves between pre-intervention and post-intervention $(\mathrm{p}=0.033)$, indicating a more dynamic diurnal cortisol rhythm in response to the intervention. In both studies, engagement was high for all participants throughout both 6-week periods. Together, these studies highlight that it is feasible to transport people living with dementia to local galleries and museums, and that specifically tailored programming has a range of benefits. However, notably, both studies were lacking control groups and participants were selected based on their likelihood to enjoy and derive benefits from the programme.

The longest included study was a randomised controlled trial of a 5-month intergenerational mentorship programme, which included 15 people living with dementia and students at an intergenerational school. ${ }^{16}$ The school was one of the first educational programmes to enable people living with dementia to provide mentorship to younger people in a structured voluntary role. Over the 5 months, the participants alternated between 1-hour visits to a kindergarten and a sixth grade classroom for a total of 20 hours. In each class, a participant worked with two to three students and engaged in intergenerational life-history reminiscence sessions. The control group engaged in a peer education seminar titled 'Successful Aging: Reclaiming Elderhood' and received take home assignments so the time commitment was equal to the intervention group. The results indicated decreased anxiety and stress in the intervention group and an increase in the control group $(\mathrm{p}=0.049)$. Despite the small sample size, this finding supports intergenerational volunteering as a meaningful activity to promote quality of life for people living with dementia, which may also help to reduce stigma of dementia through engagement with children.

The study by Field et alinvestigated associations between nine different activities, one being horse riding (equineassisted activities) at a therapeutic riding centre. ${ }^{15}$ Eight people with mild-moderate stage dementia and previous experience with horses were recruited from a single RACF; however, only six participants met criteria to be included in the analysis. The effects of horse riding were compared with a range of activities at the RACF, including watching television, games, music, during meals and physical therapy. A modified version of the Activity in Context and Time Observational Instrument was used to evaluate environment correlates of quality of life, and specific indicators related to agitation and apparent affect, with 
the latter derived from the Apparent Affect Rating Scale. Trained researchers used a hand-held computer to record observations using an instantaneous sampling strategy at 10-minute intervals for 4 hours twice per week for eight weeks. The participants travelled by bus to the riding facility each week for 8 weeks for the therapeutic horse riding intervention and were given the choice of riding, grooming, petting or observing the horses while being guided by volunteers. Levels of conversation $(p<0.001)$ and apparent affect $(\mathrm{p}=0.001)$ were improved in horse riding compared with the other activities. The ability of the participants to converse, maintain their gaze, show pleasure and participate in complex activities were more frequently observed in the horse riding activity. Based on this small study, horse riding is considered a safe and enjoyable activity to promote quality of life of people with dementia living in RACF.

A Safe Walking Programme was investigated by MacAndrew et al in seven people with severe dementia who were prone to high-intensity wandering from two RACF. ${ }^{19}$ The 3-week intervention involved supervised walks outside the RACF each weekday. The timing of the intervention was calculated to be $30 \mathrm{~min}$ before when the participants were most likely to wander (between 7:00 and 19:00) as determined using Actigraph activity monitoring. The walks were facilitated by one trained 'interventionist' around the predetermined routes in the immediate neighbourhood of the RACFs. Each 'interventionist' followed a set protocol fidelity checklist, which was completed at the end of each walk and served as the quantitative outcome measure. The checklist included the pre-walk preparation activities, the time and duration of the walk (30 min), whether the prescribed route was followed and any deviations to the protocol. In total, 86 of the $105(80 \%)$ planned walks were completed according to the protocol fidelity checklist for an average of 12.3 walks per participant out of 15 . Ninety-one per cent of the walks lasted for the full $30 \mathrm{~min}$. The Safe Walking Programme was found to be practically feasible and perceived to have benefits for people with dementia who wander. After the study, staff reported that some participants seemed to walk with more purpose and were more likely to be attracted to areas, where group activities were occurring.

Outdoor gardening was the intervention in the study by Thelander et al. ${ }^{22}$ Eight residents of RACF were recruited for 40-70-minute gardening sessions, three times per week over a 6-week period, in groups of two or three participants. Five of the participants used a walking frame. The gardening took place in a park adjacent to the RACF, and activities focused on park maintenance, including tidying gravel paths, weeding, composting and planting flowers, although there was flexibility to perform other tasks within the participants physical capabilities. Participants were observed and rated using a 'Six-Degree Independence Scale' designed specifically for this study, which evaluated the levels of assistance required to perform the activities from independent to not participating in activities. Generally, there were no fluctuations between residents' independence across the intervention, and each resident participated between 10 times and 15 times and rated between 2 (carried out activity with supervision) and 5 (extensive need for help). Baseline score on the MMSE had no significant effect on level of independence $(p=0.50)$, although more help was required in participants with more severe dementia. The study demonstrated that people with dementia living in a RACF can be safely enabled to participate in gardening activities, which have the potential to improve physical and functional capacity in small supervised groups.

\section{DISCUSSION}

There is a need for engaging activities that are enjoyable and enable social connection for people living with dementia in RACF. To the best of our knowledge, this is the first systematic review to collate the results of all studies which facilitated participation in activities within the community for people with dementia living in RACFs to leave their facility as part of an intervention. Studies of wheelchair cycling, swimming, art gallery visits, intergenerational mentorship, horse riding, walking and outdoor gardening were identified. However, due to a paucity of studies, we could not determine which interventions are the most effective in promoting well-being. Each study focused on different outcomes; however, it was demonstrated that activities outside the care setting were feasible and had the potential to offer psychological and physical benefits to people living with dementia. Thehe systematic review revealedonly two randomised controlled trials and no blinded studies. This may be due to several factors, including practical difficulties in performing large blinded studies in RACF settings, the cost to undertake this research and the need for RACF staff to be present in order to ensure the safety of the participants. The findings of the present study identify a need for further research into how these activities can be sustainably and adequately implemented for people with dementia in the RACF setting.

\section{Psychological outcomes}

Most of the studies in this review included a psychological outcome, and demonstrated the potential for improvements in well-being, ${ }^{12-14} 21$ quality of life ${ }^{14}$ mood, ${ }^{121315}$ cognition, ${ }^{14}$ and reducing depression ${ }^{12-14} 16$ and behavioural symptoms. ${ }^{19} 21$ People with dementia in RACF are relatively high users of psychotropic agents ${ }^{24}$; however, psychosocial approaches may lead to a decrease in use and benefit mental health and well-being. ${ }^{25} 26$ Visiting an art gallery was shown to be beneficial in two studies, ${ }^{1420}$ and a range of cultural and arts-based activities has been considered to have greater benefits than pharmacological treatments as they promote social functioning and can facilitate meaningful personal experiences. ${ }^{27}$ Similarly, exercise, as shown in this review through walking, swimming, gardening and horse riding, has positive effects on cognitive performance, and can 
reduce depression and anxiety. ${ }^{28}$ Being outdoors as a passenger in wheelchair cycling also has psychological benefits by providing access to fresh air, promoting conversation, memory formation and relaxation. ${ }^{12} 1329$ All included studies facilitated social connectedness as the activities were performed in small groups or with others. This was seen in the study of gardening where participants worked together to perform a civic duty. ${ }^{22}$ Gardening and horticultural therapy have been shown to have positive psychological, social and physical benefits, and benefits are compounded when undertaken in groups. ${ }^{30}$ Interestingly, in the study by MacAndrew et al, staff found that the walking programme enriched the lives of the participants, who were selected due to their wandering, and even created positive change in the walking and wandering habits of some participants. ${ }^{19}$ While dementia is a cluster of incurable conditions, social participation and engaging in activities away from the RACF has the potential to prolong quality of life and potentially reduce behavioural symptoms in the care setting.

\section{Physical outcomes}

Swimming, gardening, horse riding and walking were physical activities used in the included studies. In particular, physical benefits, in the form of grip strength and a reduction in sleep disturbances were observed as a result of the swimming ${ }^{17} 18$ and wheelchair cycling. ${ }^{12}{ }^{13}$ Grip strength is a widely used marker of whole body muscular strength, which is associated with vitality, physical function and cognitive performance. ${ }^{31} 32$ Swimming improved grip strength, ${ }^{17} 18$ but no benefits were observed for grip strength following 6 weeks of arts-based discussion groups ${ }^{14}$ suggesting that physical activity is required to see improvements. Swimming for older people has many benefits, including an increase in metabolism which can help with sleep and appetite, in addition to being a nonweight bearing form of physical activity which enables a full range of motion and joint mobility. A reduction in sleep disturbances was observed in response to wheelchair cycling. ${ }^{12}{ }^{13}$ Individualised social activities have been shown to improve sleep drive and the circadian rhythm in people with dementia, and reduce daytime sleepiness. ${ }^{33}$ It has also been demonstrated that multicomponent interventions and light therapy can improve sleep quality. ${ }^{34}$ While sleep outcomes were not assessed in the outdoor gardening study by Thelander $e t$ al, therapeutic gardens may also improve sleep-wake cycles of people with dementia. ${ }^{35}$ Benefits can be observed from simply getting outdoors to receive increased exposure to natural sunlight. ${ }^{36}$ Gardening at a local park and walking represent ideal opportunities for people with dementia to engage in physical activity in an outdoor environment. ${ }^{19} 29$ The use of gardens and green spaces in the community represents an enabling environment for people with dementia, where they can engage in meaningful activity and achieve a sense of contribution and connection with their community. ${ }^{37}$ In the broader literature, the highest quality evidence for physical interventions in dementia, such as strength training and aerobic exercise, included a social aspect, ${ }^{2}$ and it is possible that the physical interventions identified in this review (swimming, walking, gardening and horse riding) may result in more benefits because they also involved this social aspect and took place within the community setting.

\section{Physiological outcomes}

The assessment of the physiological markers in psychosocial interventions for people with dementia is a relatively new and emerging area of research. ${ }^{38}$ The study by D'Cunha et al found an improvement in the diurnal cortisol rhythm after participating in arts-based discussion groups over 6 weeks. ${ }^{14}$ Cortisol is the bodies primary stress and awakening hormone, and impairments in the diurnal rhythm in dementia are associated with lower hippocampal volume, increased agitation, decreased resiliency and greater cognitive impairment, and is associated with depression. ${ }^{14}{ }^{39}$ Decreases in skeletal muscle mass and lean body mass were observed after participation in the Watermemories Swimming Club and in the control group. ${ }^{17}$ However, a greater decrease was observed in the control group. The maintenance of muscle mass in older people may have implications for falls prevention, and swimming can potentially improve balance, gait and functional status. ${ }^{40}$ The measurement of physiological responses, such as stress hormones levels, skin responses, and heart rate variability, have been associated with wellbeing and mental health in dementia and are likely to be improved following activities outside of the RACF. ${ }^{38}$

The capacity to facilitate meaningful activities outside the care setting may require planning and resources beyond that needed for activities within the care settings. This includes the availability of adequate staff, suitable transportation, assessing the physical abilities and needs of the residents and the suitability of the activity itself. The findings from this review demonstrate that travel into the community is not required for benefits, with the wheelchair cycling, ${ }^{12}{ }^{13}$ walking ${ }^{19}$ and gardening ${ }^{22}$ studies taking place within close proximity to the RACF, making them practical and accessible for care staff and people with dementia. The studies that involved art gallery visits, ${ }^{1420}$ horse riding ${ }^{15}$ and swimming ${ }^{17} 1821$ required the participants to travel to participate in the activity which requires suitable transport and adequate storage space for mobility equipment and care staff or volunteers. None of the included studies detailed how the travel occurred, but all indicated that they incorporated volunteers or researchers in addition to regular staff. Safety of the residents is an important consideration in the feasibility of using spaces outside the RACF, and the need for supervision can restrict potential feelings of autonomy. ${ }^{7}$ For example, in the gardening study, the participants required supervision and there was difficulty in caring for the participants at the same time even though group sizes were limited to two or three. ${ }^{22}$ The size of the RACF, and the number of residents, are also factors in the potential for community-based activities to occur. ${ }^{41}$ Despite these 
potential barriers, activities in the community should be encouraged and can be enjoyed by people with dementia. It is important to weigh up the benefits of activities outside the RACF with the risks, and ensuring that people with dementia have the right to choose whether they want to take some risk to engage in meaningful activities. ${ }^{42}$

\section{Limitations}

The weakness of the included studies was primarily seen in their study design. Only two studies were performed in a randomised controlled fashion and did not perform any blinding, ${ }^{12} 1316$ and one study was a controlled trial without randomisation. ${ }^{17}$ The remainder of the studies were limited by their quasi-experimental design. ${ }^{14} 15$ 18-22 Quasi-experimental, non-randomised studies aim to stimulate and inform more rigorous interventional research, and if well conducted, can provide evidence for causal inference. ${ }^{43}$ Overall, limited conclusions can be made based on the included studies due to their sample sizes and preliminary nature. However, evidence supporting psychosocial interventions for people with dementia are inherently difficult to conduct, may have challenges associated with recruitment and informed consent and may place additional demands on staff or carers. Accordingly, this form of evidence is often the best available and can help inform the implementation of low-risk activities and interventions in the care setting. Moreover, if there is evidence that participation in an activity can provide even a modest benefit, then it should be encouraged. We are also limited by the lack of quality assessment tools that are tailored to use to evaluate the included studies, particularly for the quasi-experimental studies as there is not one recommended tool for appraisal.

\section{Future directions}

While field trips and outings frequently occur at many RACF, there is a need for more well-funded and adequately designed research to identify and overcome the barriers to implementation to enable greater participation in the community for people with dementia. The logistics and cost of transporting groups of older people and people with dementia represents a possible barrier, and research and policy work are required to determine how to maximise opportunities for people with dementia to participate safely in the community. Given that transportation represents a barrier to participation, future research should also investigate the potential to use a variety of public and community transport options, which may also include ride sharing and autonomous vehicles as a potentially safe method of transportation for people with dementia. There is also a need for systems to be in place to best use existing public spaces, such as art galleries and museums and even local parks. One of the most significant effects of engaging in activities outside of the RACF is a reduction in falls; however, this outcome was not assessed in any of the included studies. As reduction in the likelihood of falls can reduce morbidity and mortality, prospective studies are needed to determine if dementia-specific activities outside of the RACF can reduce risk. Outside activities are not suitable for all people with dementia in RACF with physical limitations or more progressed cognitive decline. Therefore, future research should investigate how meaningful activities can be experienced in novel ways, for example, through virtual reality technology, which can potentially provide people living in RACF the sensation of being outside and provide opportunity for physical activity through exergaming. ${ }^{44}$ Finally, future studies comparing activities facilitated within and outside of RACF are needed to understand whether there are additional benefits when activities are conducted outside of the care setting.

\section{CONCLUSION}

The findings of this systematic review demonstrate that there is currently limited, preliminary evidence from interventions which enable people with dementia to engage in activities outside of the RACF setting. The results suggest that activities outside of the RACF is associated with improvements in psychological and physical health. The interventions in this review included a variety of activities with varying degrees of accessibility. In order for greater participation in community, activities and programmes to be achieved for people with dementia, high-quality studies are needed to establish an evidence base and to understand barriers to implementation in the RACF setting.

\section{Twitter Nathan Martin D'Cunha @NathanMDCunha}

Contributors NMD conceived and designed the study, performed the searches and article screening, and carried out the data extraction and quality assessment, analysis and drafted the manuscript. SI helped design the study, carried out the quality assessment and commented on the manuscript. JK and AJM provided critical analysis on the manuscript. NN helped conceive and design the study, performed the searches and article screening, and commented on the manuscript.

Funding NMD is supported by a Dementia Australia Research Foundation PhD scholarship.

Competing interests None declared.

Patient and public involvement Patients and/or the public were not involved in the design, or conduct, or reporting, or dissemination plans of this research.

Patient consent for publication Not required.

Provenance and peer review Not commissioned; externally peer reviewed.

Data availability statement All data relevant to the study are included in the article or uploaded as supplementary information. The data reported in this study are the results from publicly available peer-reviewed literature. Funding bodies had no role in the inception, design, completion or publication of this work.

Open access This is an open access article distributed in accordance with the Creative Commons Attribution Non Commercial (CC BY-NC 4.0) license, which permits others to distribute, remix, adapt, build upon this work non-commercially, and license their derivative works on different terms, provided the original work is properly cited, appropriate credit is given, any changes made indicated, and the use is non-commercial. See: http://creativecommons.org/licenses/by-nc/4.0/.

\section{ORCID iDs}

Nathan Martin D'Cunha http://orcid.org/0000-0002-4616-9931

Nenad Naumovski http://orcid.org/0000-0002-2841-4497

\section{REFERENCES}

1 Beerens HC, Sutcliffe C, Renom-Guiteras A, et al. Quality of life and quality of care for people with dementia receiving long 
term institutional care or professional home care: the European RightTimePlaceCare study. J Am Med Dir Assoc 2014;15:54-61.

2 McDermott O, Charlesworth G, Hogervorst E, et al. Psychosocial interventions for people with dementia: a synthesis of systematic reviews. Aging Ment Health 2019;23:393-403.

3 Nordhausen T, Langner H, Fleischer S, et al. [Improving psychosocial health of nursing home residents: a systematic review of interventions for prevention and health promotion]. Z Evid Fortbild Qual Gesundhwes 2019;147-148:7-19.

4 de Oliveira AM, Radanovic M, de Mello PCH, et al. Nonpharmacological interventions to reduce behavioral and psychological symptoms of dementia: a systematic review. Biomed Res Int 2015;2015:1-9.

5 Lourida I, Gwernan-Jones R, Abbott R, et al. Activity interventions to improve the experience of care in hospital for people living with dementia: a systematic review. BMC Geriatr 2020;20:131.

6 Whear R, Coon JT, Bethel A, et al. What is the impact of using outdoor spaces such as gardens on the physical and mental wellbeing of those with dementia? A systematic review of quantitative and qualitative evidence. J Am Med Dir Assoc 2014;15:697-705.

7 van den Berg MEL, Winsall M, Dyer SM, et al. Understanding the barriers and Enablers to using outdoor spaces in nursing homes: a systematic review. Gerontologist 2020;60:e254-69.

8 Moher D, Liberati A, Tetzlaff J, et al. Preferred reporting items for systematic reviews and meta-analyses: the PRISMA statement. PLOS Med 2009;6:e1000097.

9 Higgins JPT, Altman DG, Gøtzsche PC, et al. The Cochrane collaboration's tool for assessing risk of bias in randomised trials. BMJ 2011:343:d5928.

10 Wells GA, Shea B, O'Connell D, et al. The Newcastle-Ottawa scale (NOS) for assessing the quality of nonrandomised studies in metaanalyses, 2019. Available: http://www.ohri.ca/programs/clinical_ epidemiology/oxford.asp

11 Avgerinos KI, Egan JM, Mattson MP, et al. Medium chain triglycerides induce mild ketosis and may improve cognition in Alzheimer's disease. A systematic review and meta-analysis of human studies. Ageing Res Rev 2020;58:101001.

12 Buettner, PhD, LRT, CTRS† L, Fitzsimmons, MSN, GNP, ARNP S, Labban, MS, PhD JD, et al. Active lifestyle for older adults with dementia: wheelchair biking versus other recreational therapy interventions. Am J of Rec Therapy 2013;12:31-40.

13 Buettner LL, Fitzsimmons S. AD-venture program: therapeutic biking for the treatment of depression in long-term care residents with dementia. Am J Alzheimers Dis Other Demen 2002;17:121-7.

14 D'Cunha NM, McKune AJ, Isbel S, et al. Psychophysiological responses in people living with dementia after an art gallery intervention: an exploratory study. J Alzheimers Dis 2019;72:549-62.

15 Fields B, Bruemmer J, Gloeckner G, et al. Influence of an EquineAssisted activities program on Dementia-Specific quality of life. Am J Alzheimers Dis Other Demen 2018;33:309-17.

16 George DR, Singer ME. Intergenerational volunteering and quality of life for persons with mild to moderate dementia: results from a 5-month intervention study in the United States. Am J Geriatr Psychiatry 2011;19:392-6.

17 Henwood T, Neville C, Baguley C, et al. Aquatic exercise for residential aged care adults with dementia: benefits and barriers to participation. Int Psychogeriatr 2017;29:1439-49.

18 Henwood T, Neville C, Baguley C, et al. Physical and functional implications of aquatic exercise for nursing home residents with dementia. Geriatr Nurs 2015;36:35-9.

19 MacAndrew M, Kolanowski A, Fielding E, et al. "Would you like to join me for a walk?" The feasibility of a supervised walking programme for people with dementia who wander. Int J Older People Nurs 2019;14:e12244.

20 MacPherson S, Bird M, Anderson K, et al. An art gallery access programme for people with dementia: 'you do it for the moment'. Aging Ment Health 2009;13:744-52.

21 Neville C, Henwood T, Beattie E, et al. Exploring the effect of aquatic exercise on behaviour and psychological well-being in people with moderate to severe dementia: a pilot study of the Watermemories swimming Club. Australas J Ageing 2014;33:124-7.
22 Thelander VB, Wahlin T-BR, Olofsson L, et al. Gardening activities for nursing home residents with dementia. Adv Physiother 2008;10:53-6.

23 Rosenberg F. The MoMA Alzheimer's project: programming and resources for making art accessible to people with Alzheimer's disease and their caregivers. Arts Health 2009;1:93-7.

24 McDerby N, Bail K, Kosari S, et al. Canaries in the coalmine: Stakeholder perspectives of medication management reviews for residents with dementia. Res Social Adm Pharm 2020;16:1220-7.

25 Kang HS, Makimoto K, Konno R, et al. Review of outcome measures in PARO robot intervention studies for dementia care. Geriatr Nurs 2020;41:207-14.

26 Birkenhäger-Gillesse EG, Kollen BJ, Achterberg WP, et al. Effects of psychosocial interventions for behavioral and psychological symptoms in dementia on the prescription of psychotropic drugs: a systematic review and meta-analyses. J Am Med Dir Assoc 2018;19:276.e1-276.e9.

27 de Medeiros K, Basting A. "Shall I compare thee to a dose of donepezil?": cultural arts interventions in dementia care research. Gerontologist 2014;54:344-53.

28 Park J, Cohen I. Effects of exercise interventions in older adults with various types of dementia: systematic review. Act Adapt Aging 2019;43:83-117.

29 McNiel P, Westphal J. Cycling without age program: the impact for residents in long-term care. West J Nurs Res 2020;42:728-35

30 Scott TL, Masser BM, Pachana NA. Positive aging benefits of home and community gardening activities: older adults report enhanced self-esteem, productive endeavours, social engagement and exercise. SAGE Open Med 2020;8:205031212090173.

31 Jang JY, Kim J. Association between handgrip strength and cognitive impairment in elderly Koreans: a population-based cross-sectional study. J Phys Ther Sci 2015;27:3911-5.

32 McGrath R, Robinson-Lane SG, Cook S, et al. Handgrip strength is associated with poorer cognitive functioning in aging Americans. $J$ Alzheimers Dis 2019;70:1187-96.

33 Richards KC, Beck C, O'Sullivan PS, et al. Effect of individualized social activity on sleep in nursing home residents with dementia. $J$ Am Geriatr Soc 2005;53:1510-7.

34 Salami O, Lyketsos C, Rao V. Treatment of sleep disturbance in Alzheimer's dementia. Int J Geriatr Psychiatry 2011;26:771-82.

35 Detweiler MB, Sharma T, Detweiler JG, et al. What is the evidence to support the use of therapeutic gardens for the elderly? Psychiatry Investig 2012;9:100-10.

36 Martin JL, Marler MR, Harker JO, et al. A multicomponent nonpharmacological intervention improves activity rhythms among nursing home residents with disrupted sleep/wake patterns. $J$ Gerontol A Biol Sci Med Sci 2007;62:67-72.

37 Mmako NJ, Courtney-Pratt H, Marsh P. Green spaces, dementia and a meaningful life in the community: a mixed studies review. Health Place 2020;63:102344

38 Thomas GEC, Crutch SJ, Camic PM, et al. Measuring physiological responses to the arts in people with a dementia. Int J Psychophysiol 2018;123:64-73.

39 Barca ML, Eldholm RS, Persson K, et al. Cortisol levels among older people with and without depression and dementia. Int Psychogeriatr 2018:1-5.

40 Fernando E, Fraser M, Hendriksen J, et al. Risk factors associated with falls in older adults with dementia: a systematic review. Physiother Can 2017;69:161-70.

41 Smit D, de Lange J, Willemse B, et al. Predictors of activity involvement in dementia care homes: a cross-sectional study. BMC Geriatr 2017;17:175.

42 Marsh P, Kelly L. Dignity of risk in the community: a review of and reflections on the literature. Health Risk Soc 2018;20:297-311.

43 Rockers PC, Røttingen J-A, Shemilt I, et al. Inclusion of quasiexperimental studies in systematic reviews of health systems research. Health Policy 2015;119:511-21.

44 D'Cunha NM, Nguyen D, Naumovski N, et al. A mini-review of virtual Reality-Based interventions to promote well-being for people living with dementia and mild cognitive impairment. Gerontology 2019;65:430-40. 